\title{
Color-Based Segmentation vs. Stereology: A Simple Comparison Between Two Semi-Automated Methods of Image Analysis for the Quantification of Collagen
}

\author{
Segmentación Basada en Colores vs. Estereología: Una Comparación Simple entre Dos \\ Métodos Semiautomáticos de Análisis de Imágenes para la Cuantificación del Colágeno
}

Paulo Salinas $^{1}$; Jorge Sanhueza ${ }^{2}$ \& Carlos Sandoval $^{3,4}$

SALINAS, P.; SANHUEZA, J. \& SANDOVAL, C. Color-based segmentation vs. stereology: a simple comparison between two semiautomated methods of image analysis for the quantification of collagen. Int. J. Morphol., 36(3):1118-1123, 2018.

SUMMARY: Image processing techniques are being widely developed for helping specialists in analysis of histological images and its application is especially useful in obtaining numerical data for the realization of the subsequent statistical analysis. The use of these methods makes the histological analysis of experts more objective and less time-consuming. In this paper we evaluate how well the quantitative methods - color-based image segmentation and stereology - agree on average, and how well they agree for the individuals when they are used to quantify type I and III collagen. Digital images of sections of Salmo salar jaws ( $5 \mu \mathrm{m}$, SiriusRed staining) were analyzed. Collagen quantification was performed by two methods in the same group of images: i) Color Based-Segmentation ( $K$-means algorithm; pixel cluster; ImageJ32 v1.51p) and ii) Stereology ( $\mathrm{V}_{\mathrm{v}} ; \mathrm{M}_{36} ;$ STEPanizer Stereological Tools). They were evaluated 200 images per group. The difference between groups and concordance was analyzed using t-Student $(p<0.05)$ and Blant Altman Comparison Method, respectively. The data analysis of average and individual assessments showed that there is concordance between two methods. In conclusion, stereology and color-based image segmentation are powerful tools which quantify collagen in histological sections.

KEY WORDS: Collagen; Stereology; Segmentation; Image Analysis; Histology.

\section{INTRODUCTION}

Currently, tissue processing and staining have been automated in the laboratory, and yet the subsequent analysis of histological images has not been done, with routine evaluations still performed by the trained eyes of a histologist. In addition, the evaluation or visual score not only implies a heavy workload, but also has inherent consequences on obtained data such as intra- and interobserver variation, resulting in low efficiency in histological analysis (Riber-Hansen et al., 2012). In recent years, with the growing demands of quantitative analysis, digital image processing techniques attract more and more attention from morphologists. One of the most interesting situations in the field of histology and histopathology is the quantitative analysis of the collagen network in normal or pathological tissue, either in cases of hepatic or cardiac fibrosis or as a simple evaluation of the connective tissue of an organ. One of the most used methods to study the collagen network is Picrosirius Red Staining. Lattouf et al. (2014) affirmed that the picrosirius network associated with morphometric image analysis "remains the most powerful method to study and quantify collagen" phrase that was discussed by Marcos et al. (2015). Subsequently, the quantification of the types of collagens present in the tissue is possible through stereology. This conventional manual planimetry method has proven its value but it is laborious, and the result depends on the skill of the observer. However, semi-quantitative and semiautomated methods of collagen quantification have been introduced, one of these is the analysis of digital images (Daunoravicius et al., 2014).

Semi-automated analysis of images is being developed for helping specialists in analysis of histological

\footnotetext{
${ }^{1}$ Institute of Biology, Faculty of Sciences, Pontificia Universidad Católica de Valparaíso, Valparaíso, Chile.

${ }^{2}$ School of Medicine, Faculty of Sciences, Universidad Mayor, Temuco, Chile.

${ }^{3}$ Graduate School, Faculty of Veterinary Sciences, Universidad Austral de Chile, Valdivia, Chile.

${ }^{4}$ Veterinary Histopathology Center, Puerto Montt, Chile.
} 
images obtained from biopsies for diagnoses and prognoses determination. It provides users with functionalities and additional benefits to simple microscopic observation, replacing the subjective and qualitative visual evaluation - which could be affected by the quality of tissue staining - by more objectives, reproducible and quantifiable digital analyzes (Potts et al., 2010; Daunoravicius et al.), these advantages are similar to those presented by the stereological method. The semiautomated analysis of images is considered a more reliable method than the simple and traditional visual evaluation, which depends to a large extent on the experience of the researcher and, in general, can not be reproduced. Numerous recent studies show experimentally that automated evaluation can be used successfully in clinical practice and biomedical research (Kayser \& Kayser, 2013; Ohlschlegel et al., 2013; Daunoravicius et al.; Webster \& Dunstan, 2014). One of the useful semi-automated analyses to perform image analysis is the Color-Based Image Segmentation using a cluster of pixels. It consists of constructing a symbolic representation of the image: the image is described as homogeneous areas according to one or several attributes chosen a priori by the researcher (Belaid \& Mourou, 2009). Color image segmentation is fundamental and critical for quantitative histological image analysis. The histological sections are segmented according to the color, shape or texture characteristics after being acquired with a high-resolution digital camera, and then classified using commonly supervised methods (Wu et al., 2015).

Several methods based on digital image processing and pattern recognition techniques have been proposed to address the problem of histological image segmentation. There are many existing approaches in literature that can be used to segment the histological image. These include methods based on clustering: $K$-Means and Mean Shift schemes based on mixed models (Chitade \& Katiyar, 2010). To validate a new technique for quantifying collagen associated with a digital algorithm, the most frequent is to compare the results obtained from the subjective evaluation of the pathologist and validate it against the best clinically accepted method, however, Daunoravicius et al. affirm this is no longer valid. Another alternative is to compare the results with a more specific and precise method. In this sense, we propose the use of stereological methods, instead of the visual impression of the histologist. It was hypothesized that there is agreement between color segmentation and stereology to determine the amount of collagen. The objective of the study was to evaluate how well the methods - color-based image segmentation and stereology - agree on average, and how well they agree for the individuals.

\section{MATERIAL AND METHOD}

Preparation of the tissue and obtaining digital images. Sections of mandibular tissue of Salmo salar were obtained and fixed in $10 \%$ formaldehyde for $72 \mathrm{~h}$ at room temperature $\left(22^{\circ} \mathrm{C}\right)$. Then, they were dehydrated in a series of alcohols, clarified in xylol and embedded in Paraplast (Paraplast Plus embedding medium; melting point: $54{ }^{\circ} \mathrm{C}$; Sigma-Aldrich Chemical Co., St Louis, MO, USA). Serial sections were made, $5 \mu \mathrm{m}$ thick, with a motorized rotary microtome Leica RM2255 (Leica Microsystems, Switzerland). Then, the sections were rehydrated, immersed in xylol $(10 \mathrm{~min})$, exposed to descending concentrations of ethanol (100\%, $96 \%, 80 \%$ and $70 \%$, all 15 s) and finally distilled water. Staining was performed using $0.1 \%$ solution of Picrosirius red in a saturated aqueous solution of picric acid for one hour at room temperature $\left(18{ }^{\circ} \mathrm{C}\right.$; Junqueira et al., 1979). Then, sections were rinsed in $0.01 \mathrm{NHCl}$ for $2 \mathrm{~min}$, dehydrated in ascending concentrations of ethanol $(70 \%$, $80 \%, 96 \%$ and $100 \%$, each $10 \mathrm{~s}$ ) and cleared in two stages in xylene, $10 \mathrm{~min}$ each one. Sections were covered with Entellan mounting medium (Merck, Darmstadt, Germany) and a glass cover slip. The mandibular tissue were observed by polarization microscopy using a Leica ${ }^{\circledR} \mathrm{DM} 5000 \mathrm{~B}$ microscope (Leica Microsystems, Switzerland) equipped with a light circular polarized composed of a simple polarizer and a filter analyzer (Leica Microsystems, Switzerland). This filter was aligned so the background in the field of view would be as dark as possible. Collagen type III (fibrillarimmature) was identified by green birefringence, whereas collagen type I (dense-mature) was identified by yellowred birefringence (Junqueira et al. 1979). Digital images (40X) of mandibular tissue fields were obtained with a Leica ${ }^{\circledR M C 170 H D}$ digital camera using Leica Acquire v3.2 software for Mac OS X (Leica Microsystems, Switzerland) and with Digital Slide Scanner (Motic Easy Scan®).

Groups and Quantification of Collagen. For the study of quantification of collagen, the same 200 images (40X; 20 fields/per slide; 2048 x 1536 pixels) were analyzed per group, which were evaluated by two different methods. i) Group 1: Color Based-Segmentation (Color Segmentation Plug In; EPFL, Lausanne, Switzerland; ImageJ32 v1.51p) (Schindelin et al., 2015). This ImageJ plugin allows a color image or a stack of color to be segmented by pixel clustering. The cluster of colors was defined manually by three users through the software interface. $K$-means clustering algorithm was used to identify collagen type I (red birefringence) and III (green birefringence) and both were considered the region of interest (ROI), separately. $K$-means is the clustering algorithm used to determine the natural spectral groupings present in a data set and it is one of the simplest unsupervised 
learning algorithms. It is a segregating technique. The role of $K$-means is divide data into k equally high-class clusters, and revenues the directory of the cluster to which it has allocated respectively remark (Fig. 1A) (Abdul Nazeer \& Sebastian, 2009; Chitade \& Katiyar). ii) Group 2: Stereology (Volume density: $\mathrm{V}_{\mathrm{v}}$. System-Test $\mathrm{M}_{36}$, STEPanizer Stereological Tools Cruz-Orive, 1993). The $\mathrm{V}_{\mathrm{V}}$ of collagen type I and III were estimated by point counting. The counting points-hits collagen were defined manually by the researcher according to the observation of collagen Type I or Type III through the software interface. The $\mathrm{M}_{36}$ test system provided by the STEPanizer Stereological Tool software was used (Tschanz et al., 2011), which has 36 test points; the test line measures $18 \mathrm{~d}$ and the test area measures $36.36 \mathrm{~d}^{2}$. The overlapping program analyzed images from the Salmo salar jaw (Fig. 1B).

Statistic Analysis. The average concordance was evaluated by comparing the average of the differences of the measurements of the individuals by means t-Student $(p<0.05)$, taking as hypothesis of nullity the no difference. To evaluate the concordance between individuals, or bias, the variability of the differences was evaluated with $95 \%$ confidence intervals (CIs) calculated as the mean difference $\pm 1.96 \times$ standard error of the differences $(0<\mathrm{CCI}<1)$ (Altman \& Bland, 1983; Bunce, 2009). The data analysis was performed using GraphPad Prism v. 7.0d for Mac OSX (GraphPad Software, San Diego CA).

\section{RESULTS}

The PicroSiurius stain confirmed the presence of collagen type I and III in the mandibular tissue of Salmo salar. The data analysis of average concordance showed that there is concordance of quantification of collagen type I (Fig. 2A) $(p=0.0511)$ between the Color-Based Segmentation (15.85 \pm 2.57; IC95\%: 14 -17.69) and Stereology (17.97 \pm 1.9 ; IC95\%: 16.60 - 19.33) methods. In addition, there is concordance of quantification collagen type III (Fig. 2B) $(p=0.9335)$ between the color segmentation (22.37 \pm 2.51 ; IC95\%: $20.56-24.17)$ and stereology $(22.46 \pm 2.28$; IC95\%: $20.82-24.09)$. Regarding the concordance between individuals during the determination of the amount of type I and III collagen, in
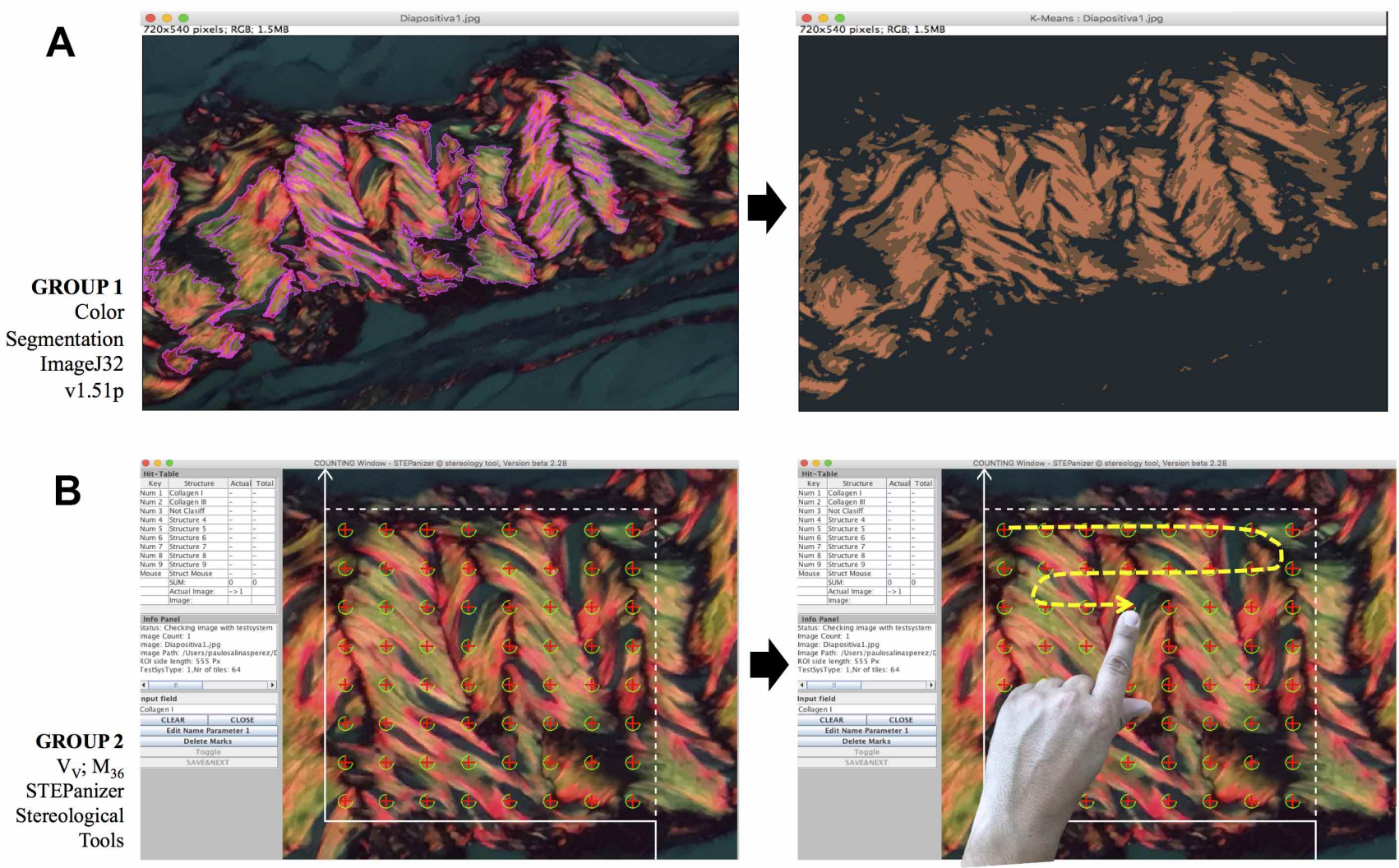

Fig. 1. A. User Interface of ImageJ. Evaluation of the amount of collagen through the application of Color-Based image analysis (Color Segmentation Plug-In; ImageJ v1.51u. National Institute of Health, USA). B. User Interface STEPanizer Stereological Tool. Evaluation of the amount of collagen through the application of Stereology through the point counting (Volume density; $\mathrm{V}_{\mathrm{v}}$ ). The counting pointshits collagen were defined manually by the researcher according to the presence of Type I or Type III through the software interface. 
both methods it was observed that $100 \%$ of the data was included within the limits of agreement (LoA). Also, it is appreciated that the difference of the observations was around 0 for most of the assessments, starting from quantities of 12 to $20 \%$ of type I collagen (Fig. 2C) and 19 to $25 \%$ of type III collagen (Fig. 2D).
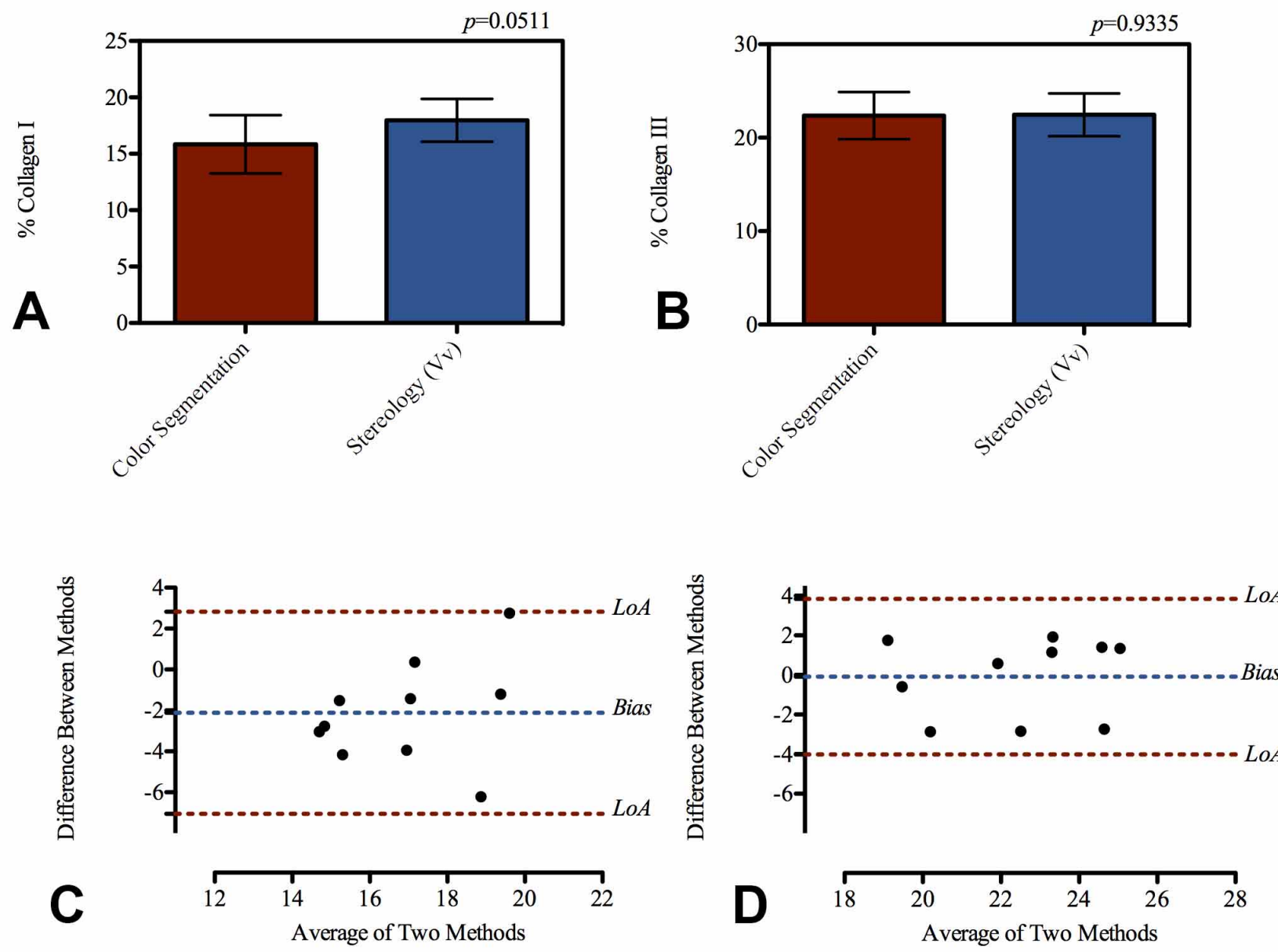

Fig. 2. Superior row: demonstrate the average of the differences of the measurements of (A) collagen type I $(p=0.0511)$ and (B) collagen type III ( $p=0.9335$ ) of the individuals by means t-Student. Inferior row: Bland-Altman plot showing the difference against the average of Color Segmentation and Stereology measurements with limits of agreement (LoA; red lines) and Bias (blue line). In (C), this plot shows the agreement between methods to quantify collagen type I, demonstrate evidence of increasing variability of differences between methods, so the LoA is much wider as the amount of collagen type I in the tissue increases. In (D), this plot shows the agreement between methods to quantify collagen type III and demonstrate no relationship between discrepancy and the level of measurement of collagen type III, so that LoA are valid and there is concordance between both methods.

\section{DISCUSSION}

Currently, the Food and Drug Administration (FDA) of the United States has approved the use of several applications of image analysis of utility in biomedicine, pathology and histology, which indicates that the semiautomated or automated quantification of histological variables provides reliable and reproducible results with greater accuracy than visual evaluation (Rojo et al., 2009). The analysis of morphometric images is very influenced by the configuration of the thresholds, the increase and the resolution of the image (Huang et al., 2013), because it is essentially a two-dimensional procedure (Marcos et al.). In contrast, stereology uses test systems that are applied to twodimensional images (as histological sections) in order to obtain the three-dimensional information that is inherent in all biological tissues (Marcos et al.). Due to the objective and independent nature of stereological methods, this method 
was used in this study as the gold standard or the reference method for comparative image analysis (Hadi et al., 2011; Daunoravicius et al.).

In our study we used one of the most used segmentation techniques for these images segmentation: the $K$-means clustering algorithm, and the objective was to evaluate the concordance in the determination of the amount of tissue collagen between two semi-automated methods frequently used in biomedical research (segmentation of image colors and stereology). Therefore, we evaluated the accuracy in obtaining data from the digital image analysis tools for the measurement of the fraction area corresponding to collagen type I and III in Salmo salar jaw biopsies stained with Picrosirius Red, considered by some researchers one of the most used methods to study and quantify the remodeling of the collagen network (Malkusch et al., 1995), and compared them with reference data obtained by stereology (point counting; $\mathrm{V}_{\mathrm{v}}$ ) in the same images.

The results obtained in this study indicate the determination of the amount of collagen based on the segmentation of colors does not differ from the results obtained by stereology, either with respect to the group average or individual variation for collagen type I and III; this considering that the standard deviation is low and, therefore, the error of variability rate is really low and stable. In addition, good agreement was observed between methods in the assessment of collagen type I and much better in that of collagen type III. Also, shows that color-based segmentation and stereology represent an important tool in biomedical research, integrating the objectiveness of the measurements, high level of reproducibility, low cost, independence of human subjectivity and possibility of quantitative analysis of the different variables. These advantages were also affirmed by Wu et al.

The assessment of the amount of collagen through the application of point counting was defined manually by the researcher according to the presence of collagen type I or type III through the software interface (Fig. 1B). Therefore, a fundamental requirement is to improve the contrast of the structures that the researcher needs to evaluate. One of the objectives of the Picrosirius Stain was to improve the contrast of the human eye to determine the intersection points of collagen in the test system during stereological study, and to determine the natural spectral groupings shown in a data set in the $K$-means.

The Picrosirius Red stain has become a common way to study the collagen network. Most images that created by polarizer and filter analyzer were completely clear and some was contain signal noise ratio (SNR), which affected collagen detection, however, we believe this did not have an effect on our results, which was demonstrated and contrasted with the stereological data. The final result of tissue recognition matches the stereology data outcome comparing with others studies using different segmentation techniques comparing with other scientific results (Varshney et al., 2009).

\section{CONCLUSIONS}

The quantification method directly influences the estimation of the amount of collagen in a tissue, so it requires particular attention, especially when comparisons between control and experimental groups are required. The present study compared two methods for assessing collagen. According to the evidence there is concordance in the quantitative data obtained through the Semi-automated Method (Color-Based Segmentation) and Stereology (point counting), which proved to be accurate and sensitive for the quantification of collagen. The results - and possibly also the intergroup comparisons - of the collagen estimation depend directly on the quantification method used. The simple application of semi-automated tools in the analysis of images as it was done here can be recommended for the quantification of collagen, however it requires to have considerations mainly avoid including in the estimation to structures that are not of interest, which would over- or underestimate the amount of collagen - dependent on tissue integrity, staining protocol and adequate image acquisition - generating erroneous results. In conclusion, stereology and color-based image segmentation are powerful tools, which can be used (combined or complementary to each other) for quantify collagen in histological sections.

SALINAS, P.; SANHUEZA, J. \& SANDOVAL, C. Segmentación basada en colores vs. estereología: Una comparación simple entre dos métodos semiautomáticos de análisis de imágenes para la cuantificación del colágeno. Int. J. Morphol., 36(3):1118-1123, 2018

RESUMEN: Las técnicas de procesamiento de imágenes han sido ampliamente desarrolladas para ayudar a los especialistas en el análisis de imágenes histológicas y su aplicación es especialmente útil para obtener datos numéricos para la realización del posterior análisis estadístico. El uso de estos métodos hace que el análisis histológico realizado por expertos sea más objetivo. En este estudio evaluamos qué tan bien coinciden o concuerdan en promedio y entre evaluaciones individuales los métodos cuantitativos "segmentación basada en color" y la "estereología" cuando son utilizados para cuantificar el colágeno tipo I y III. Se analizó imágenes digitales de tejido mandibular de Salmo salar $(5 \mu \mathrm{m}$, tinción SiriusRed). La cuantificación del colágeno se realizó me- 
diante dos métodos en el mismo grupo de imágenes: i) Segmentación basada en color (algoritmo $K$-mean, cluster de píxeles, ImageJ32 v1.51p) y ii) Estereología $\left(\mathrm{V}_{\mathrm{v}} ; \mathrm{M}_{36}\right.$; STEPanizer Stereological Tools). Fueron evaluados 200 imágenes por grupo. Se analizó la diferencia de medias y concordancia entre métodos mediante t-Students $(p>0,05)$ y el Método de Comparación de Blant-Altman. El análisis de los datos de las evaluaciones promedio e individuales demostró que hay concordancia entre los dos métodos. En conclusión, la estereología y la segmentación de imágenes basadas en color son poderosas herramientas útiles para cuantificar colágeno en secciones histológicas.

PALABRAS CLAVE: Colágeno; Estereología; Segmentación; Analisis de Imagen; Histología.

\section{REFERENCES}

Abdul Nazeer, K. A. \& Sebastian, M. P. Improving the Accuracy and Efficiency of the K-means Clustering Algorithm London, Proceedings of the World Congress on Engineering 2009 Vol I WCE 2009, July 1 3, 2009.

Altman, D. G. \& Bland, J. M. Measurement in medicine: the analysis of method comparison studies. Statistician, 32(3):307-17, 1983.

Belaid, L. J. \& Mourou, W. Image segmentation: a watershed transformation algorithm. Image Anal. Stereol., 28(2):93-102, 2009.

Bunce, C. Correlation, agreement, and Bland-Altman analysis: statistical analysis of method comparison studies. Am. J. Ophthalmol., 148(1):46, 2009.

Chitade, A. Z. \& Katiyar, S. K. Colour based image segmentation using kmeans clustering. Int. J. Eng. Sci. Technol., 2(10):5319-5325, 2010.

Cruz-Orive, L. M. Systematic sampling in stereology. Proceedings of the 49th Session of the International Statistical Institute (Book 2), 451-68, 1993.

Daunoravicius, D.; Besusparis, J.; Zurauskas, E.; Laurinaviciene, A.; Bironaite, D.; Pankuweit, S.; Plancoulaine, B.; Herlin, P.; Bogomolovas, J.; Grabauskiene, V. \& Laurinavicius, A. Quantification of myocardial fibrosis by digital image analysis and interactive stereology. Diagn. Pathol., 9:114, 2014.

Hadi, A. M.; Mouchaers, K. T.; Schalij, I.; Grunberg, K.; Meijer, G. A.; Vonk-Noordegraaf, A.; van der Laarse, W. J. \& Beliën, J. A. Rapid quantification of myocardial fibrosis: a new macro-based automated analysis. Cell. Oncol. (Dordr.), 34(4):343-54, 2011.

Huang, Y.; de Boer, W. B.; Adams, L. A.; MacQuillan, G.; Rossi, E.; Rigby, P.; Raftopoulos, S. C.; Bulsara, M. \& Jeffrey, G. P. Image analysis of liver collagen using sirius red is more accurate and correlates better with serum fibrosis markers than trichrome. Liver Int., 33(8):1249-56, 2013.

Kayser, G. \& Kayser, K. Quantitative pathology in virtual microscopy: history, applications, perspectives. Acta Histochem., 115(6):527-32, 2013.

Lattouf, R.; Younes, R.; Lutomski, D.; Naaman, N.; Godeau, G.; Senni, K. \& Changotade, S. Picrosirius red staining: a useful tool to appraise collagen networks in normal and pathological tissues. J. Histochem. Cytochem., 62(10):751-8, 2014.

Malkusch, W.; Rehn, B. \& Bruch, J. Advantages of Sirius Red staining for quantitative morphometric collagen measurements in lungs. Exp. Lung Res., 21(1):67-77, 1995.

Marcos, R.; Bragança, B. \& Fontes-Sousa, A. P. Image analysis or stereology: which to choose for quantifying fibrosis? J. Histochem. Cytochem., 63(9):734-6, 2015.

Ohlschlegel, C.; Kradolfer, D.; Hell, M. \& Jochum, W. Comparison of automated and manual FISH for evaluation of HER2 gene status on breast carcinoma core biopsies. B. M. C. Clin. Pathol., 13:13, 2013.

Potts, S. J.; Young, G. D. \& Voelker, F. A. The role and impact of quantitative discovery pathology. Drug Discov. Today, 15(21-22):943-50, 2010.

Riber-Hansen, R.; Vainer, B. \& Steiniche, T. Digital image analysis: a review of reproducibility, stability and basic requirements for optimal results. A. P. M. I. S., 120(4):276-89, 2012.

Rojo, M. G.; Bueno, G. \& Slodkowska, J. Review of imaging solutions for integrated quantitative immunohistochemistry in the Pathology daily practice. Folia Histochem. Cytobiol., 47(3):349-54, 2009.

Schindelin, J.; Rueden, C. T.; Hiner, M. C. \& Eliceiri, K. W. The ImageJ ecosystem: An open platform for biomedical image analysis. Mol. Reprod. Dev., 82(7-8):518-29, 2015.

Tschanz, S. A.; Burri, P. H. \& Weibel, E. R. A simple tool for stereological assessment of digital images: the STEPanizer. J. Microsc., 243(1):4759, 2011.

Varshney, S. S.; Rajpal, N. \& Purwar, R. Comparative study of image segmentation techniques and object matching using segmentation. Delhi, 2009 Proceeding of International Conference on Methods and Models in Computer Science (ICM2CS), 2009. pp.1-6.

Webster, J. D. \& Dunstan, R. W. Whole-slide imaging and automated image analysis: considerations and opportunities in the practice of pathology. Vet. Pathol., 51(1):211-23, 2014.

Wu, G.; Zhao, X.; Luo, S. \& Shi, H. Histological image segmentation using fast mean shift clustering method. Biomed. Eng. Online, 14:24, 2015.

Corresponding author:

Paulo Salinas

Instituto de Biología

Facultad de Ciencias

Pontificia Universidad Católica de Valparaíso

Valparaiso

CHILE

Email: paulo.salinas@pucv.cl

Recibido : 29-04-2018

Aceptado: 26-06-2018 